PROCEEDINGS OF THE

AMERICAN MATHEMATICAL SOCIETY

Volume 135, Number 6, June 2007, Pages 1785-1793

S 0002-9939(06)08814-9

Article electronically published on December 27, 2006

\title{
THE SCHRÖDINGER EQUATION WITH A MOVING POINT INTERACTION IN THREE DIMENSIONS
}

\author{
ANDREA POSILICANO
}

(Communicated by David S. Tartakoff)

\begin{abstract}
In the case of a single point interaction we improve, by using different methods, the existence theorem for the unitary evolution generated by a Schrödinger operator with moving point interactions obtained by Dell'Antonio, Figari and Teta.
\end{abstract}

\section{INTRODUCTION}

Let us denote by $L^{2}\left(\mathbb{R}^{3}\right)$, with the usual scalar product $\langle\cdot, \cdot\rangle_{2}$ and corresponding norm $\|\cdot\|_{2}$, the Hilbert space of square integrable measurable functions on $\mathbb{R}^{3}$. By $H^{1}\left(\mathbb{R}^{3}\right)$ and by $H^{2}\left(\mathbb{R}^{3}\right)$ we denote the usual Sobolev-Hilbert spaces

$$
\begin{aligned}
H^{1}\left(\mathbb{R}^{3}\right) & :=\left\{\psi \in L^{2}\left(\mathbb{R}^{3}\right): \nabla \psi \in L^{2}\left(\mathbb{R}^{3}\right)\right\}, \\
H^{2}\left(\mathbb{R}^{3}\right) & :=\left\{\psi \in H^{1}\left(\mathbb{R}^{3}\right): \Delta \psi \in L^{2}\left(\mathbb{R}^{3}\right)\right\} .
\end{aligned}
$$

Let

$$
H \equiv-\Delta: H^{2}\left(\mathbb{R}^{3}\right) \rightarrow L^{2}\left(\mathbb{R}^{3}\right)
$$

be the self-adjoint operator giving the Hamiltonian of a free quantum particle in $\mathbb{R}^{3}$. For any $y \in \mathbb{R}^{3}$ let us consider the symmetric operator $H_{y}^{\circ}$ obtained by restricting $H$ to the subspace

$$
\left\{\psi \in H^{2}\left(\mathbb{R}^{3}\right): \psi(y)=0\right\} .
$$

Such a symmetric operator has defect indices $(1,1)$. Any of its self-adjoint extensions different from $H$ itself describes a point interaction centered at $y$. One has the following (see [1], section I.1.1 as regards $H_{\alpha, y}$, and see [9] as regards $F_{\alpha, y}$ ).

Theorem 1.1. Any self-adjoint extension of $H_{y}^{\circ}$ different from $H$ itself is given by

$$
\begin{array}{ll} 
& H_{\alpha, y}: D\left(H_{\alpha, y}\right) \rightarrow L^{2}\left(\mathbb{R}^{3}\right), \\
D\left(H_{\alpha, y}\right):= & \\
\left\{\psi \in L^{2}\left(\mathbb{R}^{3}\right): \psi(x)=\right. & \left.\psi_{\lambda}(x)+\Gamma_{\alpha}(\lambda)^{-1} \psi_{\lambda}(y) \mathcal{G}_{\lambda}(x-y), \psi_{\lambda} \in H^{2}\left(\mathbb{R}^{3}\right)\right\}, \\
& \left(H_{\alpha, y}+\lambda\right) \psi:=(H+\lambda) \psi_{\lambda},
\end{array}
$$

Received by the editors February 3, 2006.

2000 Mathematics Subject Classification. Primary 47B25, 47D08; Secondary 47D06, 81Q10.

Key words and phrases. Point interactions, singular perturbations, unitary propagators.

(C)2006 American Mathematical Society Reverts to public domain 28 years from publication 
the definition being $\lambda$-independent. Here $\alpha \in \mathbb{R}$,

$$
\Gamma_{\alpha}(\lambda)=\alpha+\frac{\sqrt{\lambda}}{4 \pi}, \quad \mathcal{G}_{\lambda}(x)=\frac{e^{-\sqrt{\lambda}|x|}}{4 \pi|x|},
$$

and $\lambda>0$ is chosen in such a way that $\Gamma_{\alpha}(\lambda) \neq 0$. The kernel of the resolvent of $H_{\alpha, y}$ is given by

$$
\left(H_{\alpha, y}+\lambda\right)^{-1}\left(x_{1}, x_{2}\right)=\mathcal{G}_{\lambda}\left(x_{1}-x_{2}\right)+\Gamma_{\alpha}(\lambda)^{-1} \mathcal{G}_{\lambda}\left(x_{1}-y\right) \mathcal{G}_{\lambda}\left(x_{2}-y\right) .
$$

The quadratic form associated with $H_{\alpha, y}$ is

$$
F_{\alpha, y}: D\left(F_{y}\right) \times D\left(F_{y}\right) \rightarrow \mathbb{R}
$$

$D\left(F_{y}\right):=$

$$
\begin{gathered}
\left\{\psi \in H^{1}\left(\mathbb{R}^{3}\right): \psi(x)=\psi_{\lambda}(x)+q_{\psi} \mathcal{G}_{\lambda}(x-y), \psi_{\lambda} \in H^{1}\left(\mathbb{R}^{3}\right), q_{\psi} \in \mathbb{C}\right\}, \\
\left(F_{\alpha, y}+\lambda\right)(\psi, \phi)=\left\langle\nabla \psi_{\lambda}, \nabla \phi_{\lambda}\right\rangle_{2}+\lambda\left\langle\psi_{\lambda}, \phi_{\lambda}\right\rangle_{2}+\Gamma_{\alpha}(\lambda) \bar{q}_{\psi} q_{\phi} .
\end{gathered}
$$

Moreover the essential spectrum of $H_{\alpha, y}$ is purely absolutely continuous,

$$
\begin{gathered}
\sigma_{e s s}\left(H_{\alpha, y}\right)=\sigma_{a c}\left(H_{\alpha, y}\right)=[0, \infty), \\
\alpha<0 \Rightarrow \sigma_{p p}\left(H_{\alpha, y}\right)=-(4 \pi \alpha)^{2}, \\
\alpha \geq 0 \Rightarrow \sigma_{p p}\left(H_{\alpha, y}\right)=\emptyset .
\end{gathered}
$$

Suppose now that the point $y$ is not fixed but describes a curve $y: \mathbb{R} \rightarrow \mathbb{R}^{3}$, thus producing the family of self-adjoint operators $H_{\alpha, y}(t) \equiv H_{\alpha, y(t)}$. Then one is interested in showing that the time-dependent Hamiltonian $H_{\alpha, y}(t)$ generates a strongly continuous unitary propagator $U_{t, s}$. Note that both the operator and the form domain of $H_{\alpha, y}(t)$ are strongly time dependent. This renders inapplicable the known general theorems (see [3, 6]), and such a generation problem is not trivial.

By exploiting the explicit form of $H_{\alpha, y}(t)$ and in the case of several moving point interactions, Dell'Antonio, Figari and Teta obtained in 2] the following (here we state their results in the simpler case of a single point interaction).

Theorem 1.2. Suppose that

$$
y \in C^{3}\left(\mathbb{R} ; \mathbb{R}^{3}\right), \quad \psi \in C_{0}^{\infty}\left(\mathbb{R}^{3}\right), \quad \psi(y(s))=0 .
$$

Then there exists a unique strongly continuous unitary propagator

$$
U_{t, s}: L^{2}\left(\mathbb{R}^{3}\right) \rightarrow L^{2}\left(\mathbb{R}^{3}\right)
$$

such that

$$
\left(i \frac{d}{d t} U_{t, s} \psi, \phi\right)_{t}=F_{\alpha, y}(t)\left(U_{t, s} \psi, \phi\right)
$$

for all $\phi \in D\left(F_{y}(t)\right)$.

Here $(\cdot, \cdot)_{t}$ denotes the duality between $D\left(F_{y}(t)\right)$ and its strong dual. Moreover the solution $\psi(t):=U_{t, s} \psi$ has a natural representation (see 2, (14)-(21) for the details).

In the introduction of 2] the authors conjectured that $U_{t, s}$ defines a flow on $D\left(F_{y}(t)\right)$ which is continuous with respect to the Banach topology induced by the quadratic form $F_{\alpha, y}(t)$.

Here we show, by using different methods, that if $y \in C^{2}\left(\mathbb{R} ; \mathbb{R}^{3}\right)$, then this is indeed the case, and Theorem 1.2 above holds for any $\psi \in D\left(F_{y}(s)\right.$ ) (see Theorem 3.1 for the precise statements). 
Our proof proceeds in the following conceptually simple way. Noting that the unitary map

$$
T_{t} \psi(x):=\psi(x+y(t))
$$

transforms the equation

$$
i \frac{d \psi}{d t}(t)=H \psi(t)
$$

into the nonautonomous equation

$$
i \frac{d \psi}{d t}(t)=H_{\mathrm{v}}(t) \psi(t) \equiv(H+i \mathrm{v}(t) \cdot \nabla) \psi(t), \quad \mathrm{v}(t) \equiv \frac{d y}{d t}(t),
$$

we consider the point perturbations (at $y=0$ ) of $H_{\mathrm{v}}$, where $\mathrm{v}$ is a given, timeindependent vector in $\mathbb{R}^{3}$. We realize (see Theorem 2.3 ) that the form domains $D\left(F_{\mathrm{v}}\right)$ of such singular perturbations $H_{\mathrm{v}, \alpha}$ of $H_{\mathrm{v}}$ are $\mathrm{v}$-independent. Indeed one has $D\left(F_{\mathrm{v}}\right) \equiv D\left(F_{0}\right)$, where $D\left(F_{0}\right)$ is the form domain of $H_{\alpha, y}, y=0$. This allows, in the case the vector $\mathrm{v}$ is time-dependent, the application of Kisyński's theorem (see the Appendix), thus obtaining a strongly continuous unitary propagator $\tilde{U}_{t, s}$ which is also a strongly continuous propagator on $D\left(F_{0}\right)$ with respect to the Banach topology induced by the quadratic form associated with $H_{\alpha, y}, y=0$. Moreover $F_{\mathrm{v}, \alpha}$, the quadratic form associated with $H_{\mathrm{v}, \alpha}$, and $F_{\alpha, 0}$, the quadratic form associated with $H_{\alpha, 0}$, are related by the identity (see Theorem 2.3] again)

$$
F_{\mathrm{v}, \alpha}=F_{\alpha, 0}+Q_{\mathrm{v}},
$$

where $Q_{\mathrm{v}}$ is the quadratic form associated with the natural extension of $i \mathrm{v} \cdot \nabla$ to $D\left(F_{0}\right)$ (see Remark 2.4). This allows us to show (see Theorem 3.1) that

$$
U_{t, s}:=T_{t}^{-1} \tilde{U}_{t, s} T_{s}
$$

satisfies (1.1) for any $\psi \in D\left(F_{\alpha, y}(s)\right)$ and is a continuous flow from $D\left(F_{\alpha, y}(s)\right)$ onto $D\left(F_{\alpha, y}(t)\right)$. In the case $y \in C^{3}\left(\mathbb{R} ; \mathbb{R}^{3}\right)$ we also show that $U_{t, s}$ maps $\tilde{D}\left(H_{\alpha, y}(s)\right)$ onto $\tilde{D}\left(H_{\alpha, y}(t)\right)$, where

$$
\tilde{D}\left(H_{\alpha, y}(t)\right):=V_{t} D\left(H_{\alpha, y}(t)\right), \quad V_{t} \psi(x):=e^{i \mathrm{v}(t) \cdot x / 2} \psi(x) .
$$

\section{The OpERATOR $-\Delta+i L_{\mathrm{v}}$ With A POINT INTERACTION}

Let us consider the linear operator $-\Delta+i L_{\mathrm{v}}$, where

$$
L_{\mathrm{v}} \psi:=\sum_{k=1}^{3} \mathrm{v}_{k} \partial_{k} \psi, \quad \mathrm{v} \equiv\left(\mathrm{v}_{1}, \mathrm{v}_{2}, \mathrm{v}_{3}\right) \in \mathbb{R}^{3}
$$

Since, for any $\epsilon>0$,

$$
\begin{aligned}
\left\|L_{\mathrm{v}} \psi\right\|_{2}^{2} & \leq \sum_{1 \leq k, j \leq 3}\left|\mathrm{v}_{k} \mathrm{v}_{j}\left\langle\partial_{k j}^{2} \psi, \psi\right\rangle_{2}\right| \leq 3|\mathrm{v}|^{2}\left|\langle\Delta \psi, \psi\rangle_{2}\right| \\
& \leq \frac{3}{2}|\mathrm{v}|^{2}\left(\epsilon\|\Delta \psi\|_{2}^{2}+\frac{1}{\epsilon}\|\psi\|_{2}^{2}\right),
\end{aligned}
$$

by Kato-Rellich's theorem one has that

$$
H_{v}:=-\Delta+i L_{v}: H^{2}\left(\mathbb{R}^{3}\right) \rightarrow L^{2}\left(\mathbb{R}^{3}\right)
$$

is self-adjoint. Moreover, since, for any $\epsilon>0$,

$$
\left|\left\langle L_{\mathrm{v}} \psi, \psi\right\rangle_{2}\right| \leq|\mathrm{v}|\|\nabla \psi\|_{2}\|\psi\|_{2} \leq \frac{|\mathrm{v}|}{2}\left(\epsilon\|\nabla \psi\|_{2}^{2}+\frac{1}{\epsilon}\|\psi\|_{2}^{2}\right),
$$


$H_{\mathrm{v}}$ has lower bound $-|\mathrm{v}|^{2} / 4$.

Now we look for the self-adjoint extensions of the symmetric operator $H_{\mathrm{v}}^{\circ}$ obtained by restricting $H_{\mathrm{v}}$ to the kernel of the continuous and surjective linear map

$$
\tau: H^{2}\left(\mathbb{R}^{3}\right) \rightarrow \mathbb{C}, \quad \tau \psi:=\psi(0) .
$$

Theorem 2.1. Any self-adjoint extension of $H_{\mathrm{v}}^{\circ}$ different from $H_{\mathrm{v}}$ itself is given by

$$
\begin{gathered}
H_{\mathrm{v}, \alpha}: D\left(H_{\mathrm{v}, \alpha}\right) \rightarrow L^{2}\left(\mathbb{R}^{3}\right), \\
D\left(H_{\mathrm{v}, \alpha}\right):=\left\{\psi \in L^{2}\left(\mathbb{R}^{3}\right): \psi=\psi_{\lambda}+\Gamma_{\mathrm{v}, \alpha}(\lambda)^{-1} \psi_{\lambda}(0) \mathcal{G}_{\lambda}^{\mathrm{v}}, \psi_{\lambda} \in H^{2}\left(\mathbb{R}^{3}\right)\right\}, \\
\left(H_{\mathrm{v}, \alpha}+\lambda\right) \psi:=\left(H_{\mathrm{v}}+\lambda\right) \psi_{\lambda},
\end{gathered}
$$

the definition being $\lambda$-independent. Here $\alpha \in \mathbb{R}$,

$$
\Gamma_{\mathrm{v}, \alpha}(\lambda)=\alpha+\frac{\sqrt{\lambda-|\mathrm{v}|^{2} / 4}}{4 \pi}, \quad \mathcal{G}_{\lambda}^{\vee}(x)=\frac{e^{-\sqrt{\lambda-|\mathrm{v}|^{2} / 4}|x|}}{4 \pi|x|} e^{i \mathrm{v} \cdot x / 2},
$$

and $\lambda>|\mathrm{v}|^{2} / 4$ is chosen in such a way that $\Gamma_{\mathrm{v}, \alpha}(\lambda) \neq 0$. The kernel of the resolvent of $H_{\mathrm{v}, \alpha}$ is given by

$$
\left(H_{\mathrm{v}, \alpha}+\lambda\right)^{-1}\left(x_{1}, x_{2}\right)=\mathcal{G}_{\lambda}^{\mathrm{v}}\left(x_{1}-x_{2}\right)+\Gamma_{\mathrm{v}, \alpha}(\lambda)^{-1} \mathcal{G}_{\lambda}^{\mathrm{v}}\left(x_{1}\right) \mathcal{G}_{\lambda}^{-\mathrm{v}}\left(x_{2}\right) .
$$

Moreover the spectrum of $H_{\mathrm{v}, \alpha}$ is purely absolutely continuous,

$$
\begin{gathered}
\sigma_{e s s}\left(H_{\mathrm{v}, \alpha}\right)=\sigma_{a c}\left(H_{\mathrm{v}, \alpha}\right)=\left[-|\mathrm{v}|^{2} / 4, \infty\right), \\
\alpha<0 \Rightarrow \sigma_{p p}\left(H_{\mathrm{v}, \alpha}\right)=-(4 \pi \alpha)^{2}-|\mathrm{v}|^{2} / 4, \\
\alpha \geq 0 \Rightarrow \sigma_{p p}\left(H_{\mathrm{v}, \alpha}\right)=\emptyset .
\end{gathered}
$$

Proof. Let us define the bounded linear operators

$$
\breve{G}(\lambda): L^{2}\left(\mathbb{R}^{3}\right) \rightarrow \mathbb{C}, \quad \breve{G}(\lambda):=\tau\left(H_{\vee}+\lambda\right)^{-1},
$$

and

$$
G(\lambda): \mathbb{C} \rightarrow L^{2}\left(\mathbb{R}^{3}\right), \quad G(\lambda):=\breve{G}(\lambda)^{*} .
$$

Since

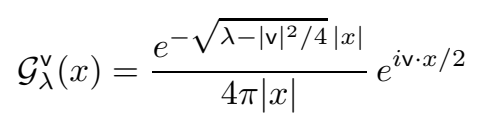

is the Green function of $H_{\mathrm{v}}+\lambda$ (see e.g. [5]), one obtains

$$
\breve{G}(\lambda) \psi=\left\langle\mathcal{G}_{\lambda}^{-v}, \psi\right\rangle_{2}, \quad G(\lambda) q=q \mathcal{G}_{\lambda}^{\vee} .
$$

Since (see [7, Lemma 2.1)

$$
(\mu-\lambda)\left(H_{\mathrm{v}}+\lambda\right)^{-1} G(\mu)=G(\lambda)-G(\mu),
$$

one obtains (see [7, Lemma 2.2)

$$
\begin{aligned}
& (\mu-\lambda) \breve{G}(\lambda) G(\mu) \\
= & \tau(G(\lambda)-G(\mu))=\tau(G(\nu)-G(\mu))-\tau(G(\nu)-G(\lambda)) \\
= & \frac{\sqrt{\mu-|\mathrm{v}|^{2} / 4}}{4 \pi}-\frac{\sqrt{\nu-|\mathrm{v}|^{2} / 4}}{4 \pi}-\left(\frac{\sqrt{\lambda-|\mathrm{v}|^{2} / 4}}{4 \pi}-\frac{\sqrt{\nu-|\mathrm{v}|^{2} / 4}}{4 \pi}\right) \\
= & \frac{\sqrt{\mu-|\mathrm{v}|^{2} / 4}}{4 \pi}-\frac{\sqrt{\lambda-|\mathrm{v}|^{2} / 4}}{4 \pi} .
\end{aligned}
$$


The thesis about $H_{\mathrm{v}, \alpha}$ and its resolvent then follows from Theorem 2.1 in [7]. As regards the spectral properties of $H_{\mathrm{v}, \alpha}$ one proceeds as in [1, Theorem 1.1.4 .

Remark 2.2. Note that, as expected, $H_{\mathrm{v}, \alpha}$ converges in norm resolvent sense to $H_{\alpha, 0}$ as $|\mathrm{v}| \downarrow 0$.

Theorem 2.3. The quadratic form associated with $H_{\mathrm{v}, \alpha}$ is

$$
F_{\mathrm{v}, \alpha}: D\left(F_{0}\right) \times D\left(F_{0}\right) \rightarrow \mathbb{R}, \quad F_{\mathrm{v}, \alpha}=F_{\alpha, 0}+Q_{\mathrm{v}}
$$

where $D\left(F_{0}\right)$ is the domain of the quadratic form $F_{\alpha, 0}$ associated with $H_{\alpha, y}, y=0$, (see Theorem 1.1) and

$$
\begin{gathered}
Q_{\mathrm{v}}: D\left(F_{0}\right) \times D\left(F_{0}\right) \rightarrow \mathbb{R} \\
Q_{\mathrm{v}}(\psi, \phi):=\left\langle i L_{\mathrm{v}} \psi_{\lambda}, \phi_{\lambda}\right\rangle_{2}+\bar{q}_{\psi}\left\langle\mathcal{G}_{\lambda}, i L_{\mathrm{v}} \phi_{\lambda}\right\rangle_{2}+q_{\phi}\left\langle i L_{\mathrm{v}} \psi_{\lambda}, \mathcal{G}_{\lambda}\right\rangle_{2} .
\end{gathered}
$$

Proof. Given $\psi$ and $\phi$ in $D\left(H_{\mathrm{v}, \alpha}\right)$ put

$$
q_{\psi}:=\Gamma_{\mathrm{v}, \alpha}(\lambda)^{-1} \psi_{\lambda}(0), \quad q_{\phi}:=\Gamma_{\mathrm{v}, \alpha}(\lambda)^{-1} \phi_{\lambda}(0) .
$$

Then

$$
\begin{aligned}
\left\langle\left(H_{\mathrm{v}, \alpha}+\lambda\right) \psi, \phi\right\rangle_{2} & =\left\langle\left(H_{\mathrm{v}}+\lambda\right) \psi_{\lambda}, \phi_{\lambda}\right\rangle_{2}+q_{\phi}\left\langle\left(H_{\mathrm{v}}+\lambda\right) \psi_{\lambda}, \mathcal{G}_{\lambda}^{\vee}\right\rangle_{2} \\
& =\left\langle\left(H_{\mathrm{v}}+\lambda\right) \psi_{\lambda}, \phi_{\lambda}\right\rangle_{2}+\Gamma_{\mathrm{v}, \alpha}(\lambda) \bar{q}_{\psi} q_{\phi} .
\end{aligned}
$$

Thus one is lead to define the quadratic form

$$
F_{\mathrm{v}, \alpha}: D\left(F_{\mathrm{v}}\right) \times D\left(F_{\mathrm{v}}\right) \rightarrow \mathbb{R}
$$

by

$$
\begin{aligned}
D\left(F_{\mathrm{v}}\right):=\left\{\psi \in H^{1}\left(\mathbb{R}^{3}\right): \psi=\psi_{\lambda}+q_{\psi} \mathcal{G}_{\lambda}^{\mathrm{v}}, \quad \psi_{\lambda} \in H^{1}\left(\mathbb{R}^{3}\right), q_{\psi} \in \mathbb{C}\right\}, & \\
& \left(F_{\mathrm{v}, \alpha}+\lambda\right)(\psi, \phi) \\
:= & \left\langle\left(-\Delta+i L_{\mathrm{v}}+\lambda\right)^{1 / 2} \psi_{\lambda},\left(-\Delta+i L_{\mathrm{v}}+\lambda\right)^{1 / 2} \phi_{\lambda}\right\rangle_{2}+\Gamma_{\mathrm{v}, \alpha}(\lambda) \bar{q}_{\psi} q_{\phi} .
\end{aligned}
$$

It is then straightforward to check that $F_{\mathrm{v}, \alpha}$ is closed and bounded from below. Thus $F_{\mathrm{v}, \alpha}$ is the quadratic form associated with $H_{\mathrm{v}, \alpha}$. Since

$$
\mathcal{G}_{\lambda}^{\vee}-\mathcal{G}_{\lambda} \in H^{1}\left(\mathbb{R}^{3}\right)
$$

one obtains $D\left(F_{\mathrm{v}}\right) \equiv D\left(F_{0}\right)$. Re-writing the quadratic form above by using the decomposition entering in the definition of $D\left(F_{0}\right)$ and noting that

$$
\forall \psi \in H^{1}\left(\mathbb{R}^{3}\right) \quad\left(F_{\vee}+\lambda\right)\left(\mathcal{G}_{\lambda}^{\vee}-\mathcal{G}_{\lambda}, \psi\right)=\left\langle\mathcal{G}_{\lambda}, i L_{\mathrm{v}} \psi\right\rangle_{2},
$$

one obtains

$$
\begin{aligned}
& \left(F_{\mathrm{v}, \alpha}+\lambda\right)(\psi, \phi)=\left\langle\nabla \psi_{\lambda}, \nabla \phi_{\lambda}\right\rangle_{2}+\lambda\left\langle\psi_{\lambda}, \phi_{\lambda}\right\rangle_{2}+Q_{\mathrm{v}}(\psi, \phi) \\
& +\left(\Gamma_{\mathrm{v}, \alpha}(\lambda)+\left(F_{\mathrm{v}, \alpha}+\lambda\right)\left(\mathcal{G}_{\lambda}^{\mathrm{v}}-\mathcal{G}_{\lambda}, \mathcal{G}_{\lambda}^{\mathrm{v}}-\mathcal{G}_{\lambda}\right)\right) \bar{q}_{\psi} q_{\phi} .
\end{aligned}
$$

Since $L_{\mathrm{v}}$ is skew-adjoint one has $\left\langle L_{\mathrm{v}} \psi, \psi\right\rangle_{2}=0$ for any real-valued $\psi \in H^{1}\left(\mathbb{R}^{3}\right)$. Thus, by taking a real-valued $J_{\epsilon} \in C_{0}^{\infty}\left(\mathbb{R}^{3}\right)$ such that $J_{\epsilon}$ weakly converges to the 
Dirac mass at the origin as $\epsilon \downarrow 0$, one obtains (here $*$ denotes convolution)

$$
\begin{aligned}
& \left(F_{\mathrm{v}, \alpha}+\lambda\right)\left(\mathcal{G}_{\lambda}^{\vee}-\mathcal{G}_{\lambda}, \mathcal{G}_{\lambda}^{\vee}-\mathcal{G}_{\lambda}\right)=\left\langle\mathcal{G}_{\lambda}, i L_{\mathrm{v}}\left(\mathcal{G}_{\lambda}^{\vee}-\mathcal{G}_{\lambda}\right)\right\rangle_{2} \\
& =\lim _{\epsilon \downarrow 0}\left\langle i L_{\vee} \mathcal{G}_{\lambda} * J_{\epsilon}, \mathcal{G}_{\lambda}^{\vee}-\mathcal{G}_{\lambda} * J_{\epsilon}\right\rangle_{2}=\lim _{\epsilon \downarrow 0}\left\langle i L_{\vee} \mathcal{G}_{\lambda} * J_{\epsilon}, \mathcal{G}_{\lambda}^{\vee}\right\rangle_{2} \\
& =\lim _{\epsilon \downarrow 0}\left\langle\left(H_{\mathrm{v}}+\lambda\right)\left(\mathcal{G}_{\lambda}^{\mathrm{v}}-\mathcal{G}_{\lambda}\right) * J_{\epsilon}, \mathcal{G}_{\lambda}^{\vee}\right\rangle_{2} \\
& =\lim _{\epsilon \downarrow 0}\left\langle\mathcal{G}_{\lambda}^{\vee}-\mathcal{G}_{\lambda},\left(H_{\vee}+\lambda\right) G_{\lambda}^{\vee} * J_{\epsilon},\right\rangle_{2}=\lim _{\epsilon \downarrow 0}\left\langle\mathcal{G}_{\lambda}^{\vee}-\mathcal{G}_{\lambda}, J_{\epsilon},\right\rangle_{2} \\
& =\left(\mathcal{G}_{\lambda}^{\mathrm{v}}-\mathcal{G}_{\lambda}\right)(0)=\frac{\sqrt{\lambda}-\sqrt{\lambda-|\mathrm{v}|^{2} / 4}}{4 \pi} \equiv \Gamma_{\alpha}(\lambda)-\Gamma_{\mathrm{v}, \alpha}(\lambda),
\end{aligned}
$$

and the proof is done.

Remark 2.4. Let $J_{\epsilon}$ be a real-valued, compactly supported smooth function weakly converging to the Dirac mass a zero as $\epsilon \downarrow 0$. For any $\psi=\psi_{\lambda}+q_{\psi} \mathcal{G}_{\lambda}$ and $\phi=$ $\phi_{\lambda}+q_{\phi} \mathcal{G}_{\lambda}$, let us define $\psi_{\epsilon}:=\psi_{\lambda}+q_{\psi} \mathcal{G}_{\lambda} * J_{\epsilon}$ and $\phi_{\epsilon}:=\phi_{\lambda}+q_{\phi} \mathcal{G}_{\lambda} * J_{\epsilon}$. Then, since $L_{\mathrm{v}}$ is skew-adjoint, one has

$$
\begin{aligned}
& \lim _{\epsilon \downarrow 0}\left\langle i L_{\vee} \psi_{\epsilon}, \phi_{\epsilon}\right\rangle_{2}=\lim _{\epsilon \downarrow 0}\left(\left\langle i L_{\vee} \psi_{\lambda}, \phi_{\lambda}\right\rangle_{2}+\bar{q}_{\psi}\left\langle\mathcal{G}_{\lambda} * J_{\epsilon}, i L_{\vee} \phi_{\lambda}\right\rangle_{2}\right. \\
& \left.+q_{\phi}\left\langle i L_{\vee} \psi_{\lambda}, \mathcal{G}_{\lambda} * J_{\epsilon}\right\rangle_{2}-i \bar{q}_{\psi} q_{\phi}\left\langle L_{\vee} \mathcal{G}_{\lambda} * J_{\epsilon}, \mathcal{G}_{\lambda} * J_{\epsilon}\right\rangle_{2}\right) \\
= & \lim _{\epsilon \downarrow 0} Q_{\vee}\left(\psi_{\epsilon}, \phi_{\epsilon}\right)=Q_{\vee}(\psi, \phi) .
\end{aligned}
$$

Thus $Q_{v}$ is the natural extension to $D\left(F_{0}\right)$ of the quadratic form associated with $i L_{\mathrm{v}}$.

\section{The Schrödinger Equation With a MOVing POINT InTERACTION}

Let us now consider a differentiable curve $y: \mathbb{R} \rightarrow \mathbb{R}^{3}$ and put $\mathbf{v}(t) \equiv \frac{d y}{d t}(t)$. Thus one has the families of self-adjoint operators and associated quadratic forms

$$
\begin{gathered}
H_{\alpha, y}(t): D\left(H_{\alpha, y}(t)\right) \rightarrow L^{2}\left(\mathbb{R}^{3}\right), \\
F_{\alpha, y}(t): D\left(F_{y}(t)\right) \times D\left(F_{y}(t)\right) \rightarrow \mathbb{R}, \\
H_{\mathrm{v}, \alpha}(t): D\left(H_{\alpha, y}(t)\right) \rightarrow L^{2}\left(\mathbb{R}^{3}\right), \\
F_{\mathrm{v}, \alpha}(t): D\left(F_{0}\right) \times D\left(F_{0}\right) \rightarrow \mathbb{R} .
\end{gathered}
$$

Now we can state our main result.

Theorem 3.1. Let $y \in C^{2}\left(\mathbb{R} ; \mathbb{R}^{3}\right)$. Then there is a unique strongly continuous unitary propagator

$$
U_{t, s}: L^{2}\left(\mathbb{R}^{3}\right) \rightarrow L^{2}\left(\mathbb{R}^{3}\right), \quad(t, s) \in \mathbb{R}^{2},
$$

such that

1)

$$
U_{t, s} D\left(F_{\alpha, y}(s)\right)=D\left(F_{\alpha, y}(t)\right) ;
$$

2) each $U_{t, s}$ is strongly continuous as a map from $D\left(F_{y}(s)\right)$ onto $D\left(F_{y}(t)\right)$ with respect to the Banach topologies induced by the bounded from below closed quadratic forms $F_{\alpha, y}(s)$ and $F_{\alpha, y}(t)$ respectively;

$$
\forall \psi \in D\left(F_{y}(s)\right), \quad t \mapsto F_{\alpha, y}(t)\left(U_{t, s} \psi, U_{t, s} \psi\right) \quad \text { is in } \quad C(\mathbb{R} ; \mathbb{R}) ;
$$

$$
\forall \psi \in D\left(F_{y}(s)\right), \quad t \mapsto U_{t, s} \psi \quad \text { is in } \quad C^{1}\left(\mathbb{R} ; D\left(F_{y}(\cdot)\right)^{*}\right),
$$


where $D\left(F_{y}(t)\right)^{*}$ denotes the dual of $D\left(F_{y}(t)\right)$ with respect to the $L^{2}\left(\mathbb{R}^{3}\right)$ scalar product;

5)

$$
\forall \psi \in D\left(F_{y}(s)\right), \forall \phi \in D\left(F_{y}(t)\right), \quad\left(i \frac{d}{d t} U_{t, s} \psi, \phi\right)_{t}=F_{\alpha, y}(t)\left(U_{t, s} \psi, \phi\right),
$$

where $(\cdot, \cdot)_{t}$ denotes the duality between $D\left(F_{y}(t)\right)$ and $D\left(F_{y}(t)\right)^{*}$.

If $y \in C^{3}\left(\mathbb{R} ; \mathbb{R}^{3}\right)$, then

6)

$$
U_{t, s} \tilde{D}\left(H_{\alpha, y}(s)\right)=\tilde{D}\left(H_{\alpha, y}(t)\right)
$$

where

$$
\tilde{D}\left(H_{\alpha, y}(t)\right):=V_{t} D\left(H_{\alpha, y}(t)\right), \quad V_{t} \psi(x):=e^{i v(t) \cdot x / 2} \psi(x) .
$$

Proof. By Theorem 2.3 we have that $y \in C^{2}\left(\mathbb{R} ; \mathbb{R}^{3}\right)$ implies that

$$
\forall \psi, \phi \in D\left(F_{0}\right), \quad t \mapsto F_{\mathrm{v}, \alpha}(t)(\psi, \phi) \quad \text { is in } \quad C^{1}(\mathbb{R}) .
$$

Let $T>0$. By Kisyński's theorem (see the Appendix) applied to the family of strictly positive self-adjoint operators

$$
H_{\mathrm{v}, \alpha}(t)+\lambda, \quad t \in[-T, T], \quad \lambda>(4 \pi \min (0, \alpha))^{2}+\frac{1}{4} \sup _{t \in[-T, T]}|\mathrm{v}(t)|,
$$

one knows that $H_{\mathrm{v}, \alpha}(t), t \in[-T, T]$, generates a strongly continuous unitary propagator $\tilde{U}_{t, s}^{T},(s, t) \in[-T, T]^{2}$. By unicity if $T^{\prime}>T$, then $\tilde{U}_{s, t}^{T}=U_{s, t}^{T^{\prime}}$ for any $(s, t) \in[-T, T]^{2} \subset\left[-T^{\prime}, T^{\prime}\right]^{2}$. Thus we obtain a unique strongly continuous unitary propagator $\tilde{U}_{t, s},(s, t) \in \mathbb{R}^{2}$, generated by the family $H_{\mathrm{v}, \alpha}(t), t \in \mathbb{R}$. Such a propagator is also a strongly continuous propagator on $D\left(F_{0}\right)$ with respect to the Banach topology induced by the bounded from below closed quadratic form $F_{\alpha, 0}$.

Considering the unitary map

$$
T_{t}: L^{2}\left(\mathbb{R}^{3}\right) \rightarrow L^{2}\left(\mathbb{R}^{3}\right), \quad T_{t} \psi(x):=\psi(x+y(t)),
$$

we then define the strongly continuous unitary propagator

$$
U_{t, s}:=T_{t}^{-1} \tilde{U}_{t, s} T_{s} .
$$

Since $T_{t}$ is a bounded operator from $D\left(F_{y}(t)\right)$ onto $D\left(F_{0}\right)$, one has that $U_{t, s}$ is a bounded operator from $D\left(F_{y}(s)\right)$ onto $D\left(F_{y}(t)\right)$ with respect to the Banach topologies induced by the bounded from below closed quadratic forms $F_{\alpha, y}(s)$ and $F_{\alpha, y}(t)$, respectively. Moreover, for all $\psi \in D\left(F_{y}(s)\right)$, the map

$$
t \mapsto F_{\alpha, y}(t)\left(U_{t, s} \psi, U_{t, s} \psi\right) \equiv F_{\alpha, 0}\left(\tilde{U}_{t, s} T_{s} \psi, \tilde{U}_{t, s} T_{s} \psi\right)
$$

is continuous. Let us now show that, for all $\psi \in D\left(F_{y}(s)\right)$ and for all $\phi \in D\left(F_{y}(t)\right)$, one has

$$
\left(i \frac{d}{d t} U_{t, s} \psi, \phi\right)_{t}=F_{\alpha, y}(t)\left(U_{t, s} \psi, \phi\right) .
$$

For any $\psi \in D\left(F_{y}(s)\right)$ and $\phi \in D\left(F_{y}(t)\right)$ there exist $\tilde{\psi}$ and $\tilde{\phi}$ in $D\left(F_{0}\right)$ such that $T_{s}^{-1} \tilde{\psi}=\psi$ and $T_{t}^{-1} \tilde{\phi}=\phi$. Thus equivalently we need to show that

$$
\left(i \frac{d}{d t} T_{t}^{-1} \tilde{U}_{t, s} \tilde{\psi}, T_{t}^{-1} \tilde{\phi}\right)_{t}=F_{\alpha, y}(t)\left(T_{t}^{-1} \tilde{U}_{t, s} \tilde{\psi}, T_{t}^{-1} \tilde{\phi}\right) \equiv F_{\alpha, 0}\left(\tilde{U}_{t, s} \tilde{\psi}, \tilde{\phi}\right)
$$


Since

$$
\begin{aligned}
& \left(i \frac{d}{d t} T_{t}^{-1} \tilde{U}_{t, s} \tilde{\psi}, T_{t}^{-1} \tilde{\phi}\right)_{t}=\left(i T_{t} \frac{d}{d t} T_{t}^{-1} \tilde{U}_{t, s} \tilde{\psi}, \tilde{\phi}\right) \\
= & \left(i T_{t}\left(\frac{d}{d t} T_{t}^{-1}\right) \tilde{U}_{t, s} \tilde{\psi}, \tilde{\phi}\right)+\left(i \frac{d}{d t} \tilde{U}_{t, s} \tilde{\psi}, \tilde{\phi}\right) \\
= & \left(i T_{t}\left(\frac{d}{d t} T_{t}^{-1}\right) \tilde{U}_{t, s} \tilde{\psi}, \tilde{\phi}\right)+F_{\mathrm{v}, \alpha}(t)\left(\tilde{U}_{t, s} \tilde{\psi}, \tilde{\phi}\right),
\end{aligned}
$$

by Theorem 2.3 we need to show that

$$
\left(i T_{t} \frac{d}{d t} T_{t}^{-1} \tilde{\psi}, \tilde{\phi}\right)=-Q_{\mathrm{v}}(\tilde{\psi}, \tilde{\phi}) .
$$

This is obviously true in the case where either $\tilde{\psi}$ or $\tilde{\phi}$ is in $H^{1}\left(\mathbb{R}^{3}\right)$ and, by taking $J_{\epsilon}$ as in Remark 2.4.

$$
\begin{aligned}
& \left(T_{t} \frac{d}{d t} T_{t}^{-1} \mathcal{G}_{\lambda}, \mathcal{G}_{\lambda}\right)=\lim _{\epsilon \downarrow 0}\left\langle T_{t} \frac{d}{d t} T_{t}^{-1} \mathcal{G}_{\lambda} * J_{\epsilon}, \mathcal{G}_{\lambda} * J_{\epsilon}\right\rangle_{2} \\
= & -\lim _{\epsilon \downarrow 0}\left\langle L_{\vee} \mathcal{G}_{\lambda} * J_{\epsilon}, \mathcal{G}_{\lambda} * J_{\epsilon}\right\rangle_{2}=0 .
\end{aligned}
$$

Thus point 5 ) is proven. Point 6 ) follows from Kisyński's theorem again by noting that if $y \in C^{3}\left(\mathbb{R} ; \mathbb{R}^{3}\right)$, then $\tilde{U}_{t, s}$ maps $D\left(H_{\mathrm{v}, \alpha}(s)\right)$ onto $D\left(H_{\mathrm{v}, \alpha}(t)\right)$ and that

$$
D\left(H_{\mathrm{v}, \alpha}(t)\right) \equiv T_{t} V_{t} D\left(H_{\alpha, y}(t)\right) .
$$

\section{Appendix: KisyńsKi's theOREM}

For the reader's convenience in this Appendix we recall Kisyński's theorem. For the proof we refer to Kisyński's original paper 4] (see in particular 4, section 8; see also [8], section II.7).

Let us recall that the double family $U_{t, s},(t, s) \in\left[T_{1}, T_{2}\right]^{2}$, is said to be a strongly continuous propagator on the Hilbert space $\mathscr{H}$ if each $U_{t, s}$ is a bounded operator on $\mathscr{H}$, the map $(t, s) \mapsto U_{t, s}$ is strongly continuous, $U_{s, s}=1$ and the ChapmanKolmogorov equation

$$
U_{t, r} U_{r, s}=U_{t, s}
$$

holds. Such a propagator is then said to be unitary if each $U_{t, s}$ is unitary.

Theorem 4.1. Let $A(t), t \in\left[T_{1}, T_{2}\right]$, be a family of strictly positive self-adjoint operators on the Hilbert space $(\mathscr{H},\langle\cdot, \cdot\rangle)$ with time-independent form domain $\mathscr{H}_{+}$. Suppose that

$$
\forall \psi, \phi \in \mathscr{H}_{+} \quad t \mapsto F(t)(\psi, \phi) \quad \text { is in } \quad C^{k}\left(\left[T_{1}, T_{2}\right] ; \mathbb{R}\right),
$$

where $F(t)$ denotes the quadratic form associated with $A(t)$.

If $k=1$, then there is a unique strongly continuous unitary propagator

$$
U_{t, s}: \mathscr{H} \rightarrow \mathscr{H}, \quad(s, t) \in\left[T_{1}, T_{2}\right]^{2},
$$

such that

1)

$$
U_{t, s} \mathscr{H}_{+}=\mathscr{H}_{+}
$$


2) $U_{t, s}$ is a strongly continuous propagator on $\left(\mathscr{H}_{+},\langle\cdot, \cdot\rangle_{+}\right)$, where $\langle\cdot, \cdot\rangle_{+}$denotes any of the equivalent scalar products

$$
\langle\psi, \phi\rangle_{t,+}:=F(t)(\psi, \phi)
$$

3)

$$
\forall \psi \in \mathscr{H}_{+} \quad t \mapsto U_{t, s} \psi \quad \text { is in } \quad C^{1}\left(\left[T_{1}, T_{2}\right] ; \mathscr{H}_{-}\right) \text {, }
$$

where $\left(\mathscr{H}_{-},\langle\cdot, \cdot\rangle_{-}\right)$is the completion of $\mathscr{H}$ endowed with any of the equivalent scalar products

4)

$$
\langle\psi, \phi\rangle_{t,-}:=\left\langle A(t)^{-1 / 2} \psi, A(t)^{-1 / 2} \phi\right\rangle
$$

$$
\forall \psi, \phi \in \mathscr{H}_{+} \quad\left(i \frac{d}{d t} U_{t, s} \psi, \phi\right)=F(t)\left(U_{t, s} \psi, \phi\right),
$$

where $(\cdot, \cdot)$ denotes the duality between $\mathscr{H}_{+}$and $\mathscr{H}_{-}$. If $k=2$, then

5)

$6)$

$$
U_{t, s} D(A(s))=D(A(t))
$$

$$
\forall \psi \in D(A(s)) \quad t \mapsto U_{t, s} \psi \quad \text { is in } \quad C^{1}\left(\left[T_{1}, T_{2}\right] ; \mathscr{H}\right) \cap C\left(\left[T_{1}, T_{2}\right] ; D(A(\cdot))\right),
$$

7)

$$
\begin{gathered}
i \frac{d}{d t} U_{t, s} \psi=A(t) U_{t, s} \psi \\
\text { REFERENCES }
\end{gathered}
$$

\section{REFERENCES}

[1] Albeverio, S., Gesztesy, F., Hoegh-Krohn, R., Holden, H.: Solvable Models in Quantum Mechanics. Second edition. AMS Chelsea Publishing, Providence, RI, 2005 MR2105735 (2005g:81001)

[2] Dell'Antonio, G.F., Figari, R., Teta, A.: The Schrödinger equation with moving point interactions in three dimensions. Stochastic processes, physics and geometry: new interplays, I (Leipzig, 1999), 99-113, CMS Conf. Proc. 28, Amer. Math. Soc., Providence, RI, 2000 MR1803381 (2002g:81028)

[3] Kato, T.: Linear evolution equations of "hyperbolic" type. J. Fac. Sci. Univ. Tokyo 27 (1970), 241-258 MR0279626 (43:5347)

[4] Kisyński, J.: Sur les opérateurs de Green des problèmes de Cauchy abstraits. Studia Math. 23 (1963/1964), 285-328 MR.0161185 (28:4393)

[5] Ortner, N.: Regularisierte Faltung von Distributionen. II. Eine Tabelle von Fundamentallösungen. Z. Angew. Math. Phys. 31 (1980), 155-173 MR0576537(82e:46058b)

[6] Pazy, A.: Semigroups of Linear Operators and Applications to Partial Differential Equations. Springer, New York, 1983 MR0710486 (85g:47061)

[7] Posilicano, A.: A Kreı̆n-like formula for singular perturbations of self-adjoint operators and applications. J. Funct. Anal. 183 (2001), 109-147 MR1837534(2002m:47018)

[8] Simon, B.: Quantum Mechanics for Hamiltonians Defined as Quadratic Forms. Princeton University Press, Princeton, N. J., 1971 MR 0455975 (56:14207)

[9] Teta, A.: Quadratic forms for singular perturbations of the Laplacian. Publ. RIMS, Kyoto Univ. 26 (1990), 803-817 MR1082317 (92g:35027)

Dipartimento di Fisica e Matematica, Universitì dell'Insubria, I-22100 Como, Italy

E-mail address: posilicano@uninsubria.it 